\title{
A WEDDERBURN DECOMPOSITION FOR CERTAIN GENERALIZED RIGHT ALTERNATIVE ALGEBRAS
}

\author{
HARRY F. SMITH
}

\begin{abstract}
Finite-dimensional nonassociative algebras are considered which satisfy certain subsets of the following identities: (1) $(x, x, x)=$ 0, (2) $(w x, y, z)+(w, x,[y, z]):=w(x, y, z)+(w, y, z) x$, (3) $(w, x \cdot y, z)=$ $x \cdot(w, y, z)+y \cdot(w, x, z),(4)(x, y, z)+(y, z, x)+(z, x, y)=0$. It is first observed that nil algebras satisfying (1) and (2) are solvable. The standard Wedderburn principal theorem is then established both for algebras satisfying (1), (2) and (3) and for algebras which satisfy (2) and (4). Throughout it is assumed that the base fields have characteristic different from 2 and 3.
\end{abstract}

1. Introduction. Using the commonly accepted notation $(x, y, z)=(x y) z$ $-x(y z),[x, y]=x y-y x$, and $x \cdot y=\frac{1}{2}(x y+y x)$, we consider nonassociative algebras which satisfy certain subsets of the following identities:

(1) $(x, x, x)=0$,

(2) $(w x, y, z)+(w, x,[y, z])=w(x, y, z)+(w, y, z) x$,

(3) $(w, x \cdot y, z)=x \cdot(w, y, z)+y \cdot(w, x, z)$,

(4) $(x, y, z)+(y, z, x)+(z, x, y)=0$.

Algebras which satisfy (1) and (2) are called generalized right alternative algebras and have been studied in [4] and [5]. Algebras satisfying (1), (2) and (3) are known as generalized alternative algebras and have been studied in [2] and [6]. Algebras which satisfy (2) and (4) are called generalized $(-1,1)$ algebras and have been considered in [4].

Let $A$ be a finite-dimensional nonassociative algebra, and let $N$ be the nil radical of $A$. Following [8] we shall say that $A$ permits a Wedderburn decomposition if $A$ has a subalgebra $S$ such that $A=S+N$ and $S \cap N$ $=(0)$.

A nonassociative algebra which satisfies (2) and

(5) $(x, y, x)=0$

is called a generalized alternative algebra II. From [11] it is known that such algebras are noncommutative Jordan, while from [10] it is known that all noncommutative Jordan algebras over fields of characteristic $\neq 2$ satisfy

(6) $\left(x, y^{2}, z\right)=2 y \cdot(x, y, z)$.

Since (3) is just the linearized form of (6), and (5) clearly implies (1), it follows that generalized alternative algebras II over fields of characteristic $\neq 2$ are generalized alternative algebras in the present sense, i.e. satisfy (1), (2) and (3).

Presented to the Society, August 27, 1975; received by the editors September 3, 1975.

AMS (MOS) subject classifications (1970). Primary 17A30.

Key words and phrases. Generalized right alternative algebra, solvable, generalized alternative algebra, generalized $(-1,1)$ algebra, Wedderburn decomposition. 
In $\$ 3$ of this work it is shown that a generalized alternative algebra $A$ over a field of characteristic $\neq 2,3$ permits a Wedderburn decomposition, provided $A / N$ is separable. This removes the restrictions imposed on such algebras by Theorem 7.14 in [2], and by the previous paragraph generalizes Theorem 2.4 in [11], except for characteristic 3 . In $\$ 4$ it is established that generalized $(-1,1)$ algebras, under the same restrictions as above, likewise permit a Wedderburn decomposition. Since all $(-1,1)$ algebras are also generalized $(-1,1)$ algebras, this extends the work in [7].

2. Generalized right alternative algebras. In this section we use heavily the fact established by Theorem 3 in [5] that semiprime generalized right alternative algebras over fields of characteristic $\neq 2,3$ are right alternative. We let $A^{[1]}=A^{(1)}=A$ and define inductively $A^{[n]}=A^{[n-1]} A, A^{(n)}$ $=A^{(n-1)} A^{(n-1)} . A$ is called right nilpotent (solvable) of index $k$ if $A^{[k]}\left(A^{(k)}\right)$ $=(0)$ for some integer $k>0$.

THEOREM 1. If $A$ is a finite-dimensional, nil, right alternative algebra over a field of characteristic $\neq 2$, then $A$ is right nilpotent and consequently solvable.

Proof. That $A$ is right nilpotent comes directly from the proof of Lemma 7 in [3]. The proof that $A$ is solvable is by induction on the dimension of $A$. Since $A$ is right nilpotent, $A^{2} \neq A$. If $A^{2}=(0)$, then clearly $A$ is solvable. Otherwise, using our induction assumption $A$ contains the nonzero solvable ideal $A^{2}$ and $A / A^{2}$ is solvable, so $A$ itself is solvable by Proposition 2.2 in [9].

THEOREM 2. If $A$ is a finite-dimensional, nil, generalized right alternative algebra over a field of characteristic $\neq 2,3$, then $A$ is solvable.

Proof. The proof is by induction on the dimension of $A$. If $A$ is semiprime, then $A$ is right alternative and consequently solvable by Theorem 1 . Thus we may assume that $A$ contains a nonzero ideal $B$ such that $B^{2}=(0)$. But then $A$ contains the solvable ideal $B$ and $A / B$ is solvable by the induction assumption, so again by Proposition 2.2 in [9] $A$ itself is solvable.

THEOREM 3. If $A \neq(0)$ is a finite-dimensional, nil semisimple, generalized right alternative algebra over a field of characteristic $\neq 2,3$, then $A$ has an identity element and is the direct sum of simple ideals.

Proof. Since $A$ nil semisimple implies $A$ is semiprime, $A$ is right alternative. But then by Theorem 6 in [1] $A$ is in fact alternative. Hence by Theorem 3.10 in [9] $A$ has an identity element, and by Theorem 3.12 in [9] $A$ is the direct sum of simple ideals.

3. Generalized alternative algebras. Let $A$ be a generalized alternative algebra over a field of characteristic $\neq 2$ which has an idempotent $e \neq 1$, and suppose moreover that $A$ has a Peirce decomposition relative to $e$. Throughout we shall use the convention that $w_{i j}, x_{i j}, y_{i j}, z_{i j} \in A_{i j}$ for $i, j=0,1$. In the subsequent lemma we shall use, of ten without specific mention, the multiplication for subspaces established in Theorem 4.1 of [2]; namely $A_{i i} A_{j i} \subset A_{i j}$, $A_{i j} A_{i j} \subset A_{j i}$ for $i \neq j$, and otherwise $A_{m n} A_{s t} \subset \delta_{n s} A_{m t}$. We shall also use the 
following facts established in Lemma 4.2, 4.3 and 5.2 of [2], where again $i \neq j$ :

(i) $\left(A_{j j}+A_{j i}+A_{i j}\right)\left(A_{i i} A_{j i}\right)=0$,

(ii) $x_{i i}\left(y_{i i} z_{j i}\right)=y_{i i}\left(z_{j i} x_{i i}\right)$,

(iii) $\left(A_{i i} A_{j i}\right) A_{i j}=0$,

(iv) $x_{i j} \cdot y_{i j}=0$.

LEMMA. Let $A$ be a generalized alternative algebra over a field of characteristic $\neq 2$ which contains an idempotent $e \neq 1$. If $A$ has a Peirce decomposition relative to $e$ and $e \in A_{10} A_{01}$, then $A_{00} A_{10}=0$.

Proof. Using the multiplication for subspaces and then in turn (3), (iv) and (i), we obtain

$$
\begin{aligned}
\left(y_{10}, z_{00} w_{01}, x_{10}\right) & =2\left(y_{10}, z_{00} \cdot w_{01}, x_{10}\right) \\
& =2\left[z_{00} \cdot\left(y_{10}, w_{01}, x_{10}\right)+w_{01} \cdot\left(y_{10}, z_{00}, x_{10}\right)\right] \\
& =z_{00}\left(y_{10}, w_{01}, x_{10}\right)+\left(y_{10}, w_{01}, x_{10}\right) z_{00},
\end{aligned}
$$

whence

$$
\begin{aligned}
z_{00}\left(y_{10}, w_{01}, x_{10}\right) & =\left(y_{10}, z_{00} w_{01}, x_{10}\right)-\left(y_{10}, w_{01}, x_{10}\right) z_{00} \\
& \in A_{01} \cap A_{10}=(0) .
\end{aligned}
$$

Thus we have

$$
z_{00}\left(\left(y_{10} w_{01}\right) x_{10}\right)=z_{00}\left(y_{10}\left(w_{01} x_{10}\right)\right) .
$$

Next using (iv) and (3) we have

$$
0=\left(w_{01}, y_{10} \cdot x_{10}, z_{00}\right)=y_{10} \cdot\left(w_{01}, x_{10}, z_{00}\right)+x_{10} \cdot\left(w_{01}, y_{10}, z_{00}\right),
$$

whence

$$
\begin{aligned}
\left(w_{01}, x_{10}, z_{00}\right) y_{10}+\left(w_{01}, y_{10}, z_{00}\right) x_{10} & =-y_{10}\left(w_{01}, x_{10}, z_{00}\right)-x_{10}\left(w_{01}, y_{10}, z_{00}\right) \\
& \in A_{01} \cap A_{10}=(0) .
\end{aligned}
$$

Thus we have

$$
\left(w_{01}, x_{10}, z_{00}\right) y_{10}=-\left(w_{01}, y_{10}, z_{00}\right) x_{10} .
$$

Now (2) gives

$$
\begin{aligned}
& \left(w_{01} x_{10}, y_{10}, z_{00}\right)+\left(w_{01}, x_{10},\left[y_{10}, z_{00}\right]\right) \\
& =w_{01}\left(x_{10}, y_{10}, z_{00}\right)+\left(w_{01}, y_{10}, z_{00}\right) x_{10} .
\end{aligned}
$$

Expansion of this last equation together with some cancellation of terms yields

$$
\begin{aligned}
& \left(w_{01} x_{10}\right)\left(z_{00} y_{10}\right)=\left(\left(w_{01} x_{10}\right) y_{10}\right) z_{00}+w_{01}\left(x_{10}\left(z_{00} y_{10}\right)\right) \\
& -w_{01}\left(\left(x_{10} y_{10}\right) z_{00}\right)-\left(w_{01}, y_{10}, z_{00}\right) x_{10}=-\left(w_{01}, y_{10}, z_{00}\right) x_{10},
\end{aligned}
$$

using the multiplication for subspaces and (i). Thus we have 
(vii)

$$
\left(w_{01} x_{10}\right)\left(z_{00} y_{10}\right)=-\left(w_{01}, y_{10}, z_{00}\right) x_{10} .
$$

Using now in turn (ii), (vii) and (vi), we obtain

$$
\begin{aligned}
z_{00}\left(y_{10}\left(w_{01} x_{10}\right)\right) & =\left(w_{01} x_{10}\right)\left(z_{00} y_{10}\right)=-\left(w_{01}, y_{10}, z_{00}\right) x_{10} \\
& =\left(w_{01}, x_{10}, z_{00}\right) y_{10},
\end{aligned}
$$

or

(viii)

$$
z_{00}\left(y_{10}\left(w_{01} x_{10}\right)\right)=\left(w_{01}, x_{10}, z_{00}\right) y_{10} .
$$

Next linearization of (1) yields

$$
\begin{gathered}
0=\left(x_{10}, w_{01}, z_{00}\right)+\left(w_{01}, z_{00}, x_{10}\right)+\left(z_{00}, x_{10}, w_{00}\right) \\
+\left(x_{10}, z_{00}, w_{01}\right)+\left(z_{00}, w_{01}, x_{10}\right)+\left(w_{01}, x_{10}, z_{00}\right) \\
=\left(x_{10}, z_{00}, w_{01}\right)+\left(z_{00}, w_{01}, x_{10}\right)+\left(w_{01}, x_{10}, z_{00}\right),
\end{gathered}
$$

using the multiplication for subspaces, (i) and (iii). Thus

$$
\left(z_{00}, w_{01}, x_{10}\right)+\left(w_{01}, x_{10}, z_{00}\right)=-\left(x_{10}, z_{00}, w_{01}\right) \in A_{00} \cap A_{11}=(0),
$$

or

$$
\left(z_{00}, w_{01}, x_{10}\right)+\left(w_{01}, x_{10}, z_{00}\right)=0 .
$$

From (2) we next obtain

$$
\begin{aligned}
& \left(z_{00} y_{10}, w_{01}, x_{10}\right)+\left(z_{00}, y_{10},\left[w_{01}, x_{10}\right]\right) \\
& =z_{00}\left(y_{10}, w_{01}, x_{10}\right)+\left(z_{00}, w_{01}, x_{10}\right) y_{10} .
\end{aligned}
$$

Expansion of this last equation together with some cancellation of terms yields

$$
\begin{aligned}
\left(z_{00} y_{10}\right)\left(x_{10} w_{01}\right)= & \left(\left(z_{00} y_{10}\right) w_{01}\right) x_{10}+z_{00}\left(y_{10}\left(x_{10} w_{01}\right)\right) \\
& -z_{00}\left(\left(y_{10} w_{01}\right) x_{10}\right)-\left(z_{00}, w_{01}, x_{10}\right) y_{10} \\
= & -z_{00}\left(\left(y_{10} w_{01}\right) x_{10}\right)-\left(z_{00}, w_{01}, x_{10}\right) y_{10},
\end{aligned}
$$

using (iii) and the multiplication for subspaces. Thus we have

$$
\left(z_{00} y_{10}\right)\left(x_{10} w_{01}\right)=-z_{00}\left(\left(y_{10} w_{01}\right) x_{10}\right)-\left(z_{00}, w_{01}, x_{10}\right) y_{10} .
$$

Finally, using in turn (x), (v), (viii) and (ix), we obtain

$$
\begin{aligned}
\left(z_{00} y_{10}\right)\left(x_{10} w_{01}\right) & =-z_{00}\left(\left(y_{10} w_{01}\right) x_{10}\right)-\left(z_{00}, w_{01}, x_{10}\right) y_{10} \\
& =-z_{00}\left(y_{10}\left(w_{01} x_{10}\right)\right)-\left(z_{00}, w_{01}, x_{10}\right) y_{10} \\
& =-\left(w_{01}, x_{10}, z_{00}\right) y_{10}-\left(z_{00}, w_{01}, x_{10}\right) y_{10} \\
& =-\left[\left(w_{01}, x_{10}, z_{00}\right)+\left(z_{00}, w_{01}, x_{10}\right)\right] y_{10}=0,
\end{aligned}
$$

or

(xi)

$$
\left(z_{00} y_{10}\right)\left(x_{10} w_{01}\right)=0
$$


Suppose now that $e \in A_{10} A_{01}$, that is $e=\sum x_{10} w_{01}$. Then by (xi) we have

$$
z_{00} y_{10}=\left(z_{00} y_{10}\right) e=\left(z_{00} y_{10}\right)\left(\sum x_{10} w_{01}\right)=0 \text {. }
$$

Hence $A_{00} A_{10}=0$ as claimed. This completes the proof of the lemma.

THEOREM 4. Let $A$ be a finite-dimensional generalized alternative algebra over a field of characteristic $\neq 2$, 3. If $A / N$ is separable where $N$ is the nil radical of $A$, then A permits a Wedderburn decomposition.

Proof. The proof is by induction on the dimension of $A$. We begin by making some reductions. First, as in the associative case, we may assume the nil radical $N$ of $A$ does not properly contain any nonzero ideal of $A$, e.g. as in $\$ 1$ of [7]. Second, if $A$ is semiprime, then by the main theorem in [6] $A$ is alternative. In this case $A$ permits a Wedderburn decomposition by Theorem 3.18 in [9]. Thus we may assume $N^{2}=(0)$ and so in the standard fashion assume the base field $F$ to be algebraically closed, e.g. as in $\$ 2$ of [7]. Third, using our Theorem 3 and Lemma 2.3 in [2], which establishes $A_{e}(1)$ and $A_{e}(0)$ are subalgebras for any idempotent $e \in A$, we may apply Theorem 2.1 in [8] to assume that $A$ has an identity element 1 and that $A / N$ is simple.

Now if $1^{\prime}$ is the only idempotent in $A / N$, which as a simple generalized alternative algebra must by the main theorem in [6] be alternative, then $A / N \simeq F 1$. This follows since $F$ is algebraically closed and since there are no nodal alternative algebras. In this case $F 1$ is the desired subalgebra of $A$. Thus we may assume $A / N$ contains an idempotent $e^{\prime} \neq 1^{\prime}$, which by Lemma 2.1 in [8] lifts to an idempotent $e \neq 1$ in $A$. Furthermore, since $A$ is finitedimensional, $e$ may be assumed to be primitive.

Next let $H=(A, e, e)$. By Lemma 2 in [4] $H$ is an ideal of $A$ such that $H^{2}=(0)$. Also, $A=A^{\prime}+H$ where $A^{\prime}$ is a subalgebra of $A$ such that $A^{\prime} \cap H=(0)$. If $H \neq(0)$, then $H=N$ by our first reduction. In this case $A^{\prime}$ is the desired subalgebra of $A$. Thus we may assume $(A, e, e)=(0)$. Then since from Lemma 2.2 in [2] it is known that always $(e, A, e)=(0)$, linearization of (1) yields $(e, e, A)=(A, e, e)=(0)$. Hence it follows that $A$ has a Peirce decomposition relative to $e$.

We now have by Theorem 5.1 in [2] that $T=A_{10} A_{01}+A_{10}+A_{01}$ $+A_{01} A_{10}$ is an ideal in $A$ generated by $A_{e}\left(\frac{1}{2}\right)=A_{10}+A_{01}$. If $T \neq A$, then $T$ permits a Wedderburn decomposition by the induction assumption, and so $A$ has a Wedderburn decomposition by Theorem 2.2 in [8]. Thus we may assume $A=A_{10} A_{01}+A_{10}+A_{01}+A_{01} A_{10}$.

Finally, since $1 \in A$ so that we have both $e \in A_{10} A_{01}$ and $1-e \in A_{01} A_{10}$ as idempotents, it follows from our previous Lemma that both $A_{00} A_{10}$ $=0$ and $A_{11} A_{01}=0$. But then $A=A_{10} A_{01}+A_{10}+A_{01}+A_{01} A_{10}$ is alternative by Theorem 5.8 in [2]. Since as noted earlier $A$ permits a Wedderburn decomposition in this case, our induction and proof are now complete.

As observed in [2], nonassociative algebras satisfying (1), (2) and (3) are anti-isomorphic to nonassociative algebras satisfying (1), (3) and

(7) $([w, x], y, z)+(w, x, y z)=y(w, x, z)+(w, x, y) z$.

Also, by Theorem 3 in [12] nonassociative algebras which satisfy (1), (2) and (7) permit a Wedderburn decomposition when they are under the same restrictions as in Theorem 4. Thus we now have the following 
COROLLARY. Let $A$ be a finite-dimensional nonassociative algebra over a field of characteristic $\neq 2,3, N$ be the nil radical of $A$, and $A / N$ be separable. If $A$ satisfies (1) and any two of (2), (3) and (7), then A permits a Wedderburn decomposition.

\section{Generalized $(-1,1)$ algebras.}

THEOREM 5. Let $A$ be a finite-dimensional generalized $(-1,1)$ algebra over a field of characteristic $\neq 2$, 3. If $A / N$ is separable where $N$ is the nil radical of $A$, then $A$ permits a Wedderburn decomposition.

Proof. The proof is by induction on the dimension of $A$. If $A$ is semiprime, then by Theorem 3 in [5] $A$ is right alternative and consequently a $(-1,1)$ algebra. In this case $A$ permits a Wedderburn decomposition by the main Theorem in [7]. Also, from (21) in [4] we know that $A_{e}(1)$ and $A_{e}(0)$ are subalgebras for any idempotent $e \in A$. Thus it follows that we may make the same first three reductions as in the proof of our Theorem 4.

Now again using Theorem 3 in [5], $A / N$ as a simple generalized $(-1,1)$ algebra must be $(-1,1)$ and so in turn is associative. Since there are no nodal associative algebras, we may thus assume as in the proof of Theorem 4 that $A$ contains a primitive idempotent $e \neq 1$. Then also as in the proof of Theorem 4 , we may assume that $H=(A, e, e)=(0)$. In particular, this means that now $A=A^{\prime}$ in Lemma 2 of [4].

Suppose next that $A$ has no nonzero trivial ideals in its center. Then by Lemma 13 in [4], $I=A_{10} A_{01}+A_{10}+A_{01}+A_{01} A_{10}$ is an ideal in the nucleus of $A$. In particular, we note that $I$ is the ideal generated by $A_{e}\left(\frac{1}{2}\right)=A_{10}$ $+A_{01}$. Now if $I \neq A$, then $I$ permits a Wedderburn decomposition by the induction assumption, and so $A$ has a Wedderburn decomposition by Theorem 2.2 in [8]. On the other hand, if $I=A$, then $A$ is associative and of course permits a Wedderburn decomposition.

There only remains to consider the case when $N$ is a nonzero trivial ideal in the center of $A$. Arguing exactly as in [7], we let $A(N)=\{x \in A \mid N x=0\}$. It is clear that $A(N)$ is an ideal in $A$ and that $N \subset A(N) \subset A$. Since $A / N$ is a simple algebra with an identity element, we have $A(N)=N$. Let $n \in N$ and $x, y \in A$. Then we have

$$
n[x, y]=n x y-n y x=n x y-x n y=n x y-n x y=0,
$$

so $[x, y] \in A(N)=N$. But then $A / N$ as a nonzero, associative, commutative, simple algebra must be a field. Since this contradicts our assumption that $A / N$ contains an idempotent different from its identity element, our proof is now complete.

ACKNowledgment. This work was supported by a grant from the Madison College Program of Grants for Faculty Research.

\section{BIBLIOGRAPHY}

1. A. A. Albert, The structure of right alternative algebras, Ann. of Math. (2) 59 (1954), 408-417. MR 15, 774.

2. S. Getu and D. J. Rodabaugh, Generalizing alternative rings, Comm. Algebra 2 (1974), 35-81. MR 50 \# 4682.

3. I. R. Hentzel, $(-1,1)$ rings, Proc. Amer. Math. Soc. 22 (1969), 367-374. MR 39 \#236. 
4. I. R. Hentzel and G. M. P. Cattaneo, Generalization of right alternative rings, Trans. Amer. Math. Soc. 207 (1975), 143-161.

5. - Semi-prime generalized right alternative rings, J. Algebra (to appear).

6. - A note on generalizing alternative rings, Proc. Amer. Math. Soc. 55 (1976), 6-8.

7. I. M. Miheev, The theorem of Wedderburn on the splitting of the radical for $a(-1,1)$ algebra, Algebra and Logic 12 (1973), 298-304.

8. D. J. Rodabaugh, On the Wedderburn principal theorem, Trans. Amer. Math. Soc. 138 (1969), 343-361. MR 48 \#8578.

9. R. D. Schafer, An introduction to nonassociative algebras, Pure and Appl. Math., vol. 22, Academic Press, New York, 1966. MR 35 \#1643.

10. I. P. Sestakov, Generalized standard rings, Algebra and Logic 13 (1974), 88-103.

11. H. F. Smith, The Wedderburn principal theorem for a generalization of alternative algebras, Trans. Amer. Math. Soc. 198 (1974), 139-154. MR 50 \#4674.

12. - The Wedderburn principal theorem for generalized alternative algebras I, Trans. Amer. Math. Soc. 212 (1975), 139-148.

Department of Mathematics, Madison College, Harrisonburg, Virginia 22801 